\title{
A Local Clinical Audit on Osteoporosis Prevention and Management in Sudanese Patients Treated with Long Term Oral Glucocorticoids for Rheumatic Diseases in a Military Tertiary Hospital in West Khartoum
}

\author{
Sara Elhadari', Hani Hussein², Alnor Alagib' ${ }^{1}$ \\ ${ }^{1}$ Rheumatology Department, Armed Forces Hospital, Khartoum, Sudan \\ ${ }^{2}$ Family Medicine Department, Armed Forces Hospital, Jubail, KSA \\ Email: sara_elhadari@yahoo.com, hanihussain@hotmail.com, elagib62@hotmail.com
}

How to cite this paper: Elhadari, S., Hussein, H. and Alagib, A. (2018) A Local Clinical Audit on Osteoporosis Prevention and Management in Sudanese Patients Treated with Long Term Oral Glucocorticoids for Rheumatic Diseases in a Military Tertiary Hospital in West Khartoum. Open Journal of Internal Medicine, 8, 207-219. https://doi.org/10.4236/ojim.2018.84020

Received: September 24, 2018 Accepted: November 11, 2018 Published: November 14, 2018

Copyright $\odot 2018$ by authors and Scientific Research Publishing Inc. This work is licensed under the Creative Commons Attribution International License (CC BY 4.0).

http://creativecommons.org/licenses/by/4.0/

\begin{abstract}
Background: Glucocorticoids (GC) are considered a mainstay as well as symptomatic and disease-modifying therapy for rheumatic diseases. They are generally used to quickly dampen inflammation, reduce duration of disease flares and as a maintenance therapy. Conventionally, oral GC are to be prescribed for short periods when possible; mainly, because of their side effects when used for long periods. One of the most important drawbacks of prolonged GC therapy is the risk of bone loss (osteoporosis (OP)) and osteoporotic fragility fracture (OFF). Objective: The aim of this audit was to assess counselling, prevention and management of $\mathrm{OP}$ in patients with rheumatic conditions who are or were receiving high doses of oral GC for three months or more against standard international guidelines. Method: The audit was carried out in March/April 2018 for a period of four weeks in a busy rheumatology service in Khartoum, Sudan. A Performa was used to collect data manually from eligible patients and their outpatient medical cards retrospectively. Then, data were audited against standard guidelines. Result: Overall, the selected Centre failed to meet the audit standard of $50 \%$ of eligible patients being appropriately managed for OP/OFF according to NICE and ACR guidelines. Conclusion: Huge areas of deficiency in the practice were identified. Acknowledging socioeconomic difficulties in the area of study, simple measures, such as carrying out a fracture risk assessment, optimizing calcium and vitamin D supplements with life style modification and patient education, would have a great impact on patients treated with steroids for rheumatic diseases.
\end{abstract}




\section{Keywords}

Rheumatic Diseases, Glucocorticoids, Osteoporosis, Audit

\section{Introduction}

Prolonged use of oral Glucocorticoids (GC) is considered a major risk factor for osteoporosis (OP)) and osteoporotic fragility fracture (OFF); Corticosteroid induced osteoporosis (CIOP) is the commonest form of secondary OP with $12 \%$ of patients on long term GC experiencing a fracture [1]. The decline in bone mass is largely determined by GC therapy dose and duration with the rate of bone loss highest during the first 3 to 6 months of steroid initiation. However, the loss is reversible once GC are discontinued [2]. In Sudan, It is surprisingly common to see patients on maintenance treatment with oral GC for their rheumatic diseases because of relative affordability and availability compared to other immunosuppressant medications and biologics.

Steroid treated patients with rheumatic diseases are good candidates to investigate the overall approach of prevention and management of OP. Some rheumatic conditions such as rheumatoid arthritis (RA) are considered independent risk factors for OP especially in those with high disease activity [3] [4] [5]. Furthermore, patients with rheumatic diseases are additionally at increased risk of having other OP risk factors; for example, some rheumatic conditions often manifest in aging population and most commonly affect females, both are more likely to suffer from OP. Moreover, rheumatic diseases can predispose to other comorbidities such as diabetes, accelerated atherosclerotic diseases, liver and kidney diseases and immobility which add to OP and OFF risk. The risk can be amplified by using oral GC treatment for long periods of time.

\section{Aims and Objectives}

To determine if eligible patients with or without other osteoporotic risk factors are being assessed for OP and OFF in accordance with internationally approved guidelines, this would require:

- To examine if patients were offered information on their risk of developing osteoporosis with long term GC therapy.

- To determine if patients were encouraged to manage modifiable risk factors such as smoking, alcohol, diet and weight.

- To examine if patients were offered Fracture Risk Assessment and or dual energy x-ray absorptiometry (DEXA) scan to assess risk of osteoporosis.

- To examine if patients had been given pharmacological therapy to prevent or treat osteoporosis.

The audit will help physicians improve the service they provide for patients with rheumatic diseases as well as improving patients' education on OP risk with GC. 


\section{Method}

This retrospective audit aimed at recruiting patients from adult rheumatology outpatient clinics in a tertiary Centre for a period of four weeks, starting from 28 March 2018 to 18 April 2018. The rheumatology clinics operate on Wednesday every week; therefore, data collection was carried out once a week for a total of 4 clinics to produce this report. Eligible patients were those diagnosed with any rheumatic disease for more than 6 months and were on, or recently on, long term oral GC therapy at a dose of $7.5 \mathrm{mg}$ or more for 3 months or more. We believe that a period of six months after diagnosis was appropriate to ensure that patients were stabilized on a long-term treatment for their conditions. Eligible patients were identified by a medical specialist and a medical officer. When a patient agreed to participate, a verbal informed consent was obtained and data were collected using the data collection Performa as described below. We inspected patients' clinic cards to confirm their diagnosis and the dosage of GC and $\mathrm{OP}$ medication given if any (e.g. calcium, vitamin $\mathrm{D}$, bisphosphonate). Some eligible subjects failed to provide their outpatient cards for inspection, therefore, they were excluded data collection was completed from patients before their regular rheumatologist consultation visit in outpatient. This center is one of the busiest rheumatology services in Sudan, therefore, it was selected for the audit. During this period forty five patients were recruited.

We utilized fracture risk assessment tools such as FRAX or QFracture to form the basis of the audit Performa. Detailed questions were added regarding patients' understanding of OP risk with GC use, prevention and management of OP, socioeconomic data, other OP risk factors and the use of corticosteroids in these patients. A pilot study was done to identify any areas of deficiencies in the audit's Performa.

\subsection{Audit Criteria and Standards}

To our knowledge, there is no formal consensus on guidelines and/or recommendations on prevention and/or management of OP in steroid-treated patients of rheumatic conditions. Therefore, we resorted to using two different guidelines to set the audit standards: National institute for health and care excellence (NICE) guidance on assessing the risk of fragility fracture [6] and the 2017 American College of Rheumatology (ACR) Guidelines for prevention and treatment of glucocorticoid-induced osteoporosis [7].

- Audit standard 1: Generally, NICE recommends that adult patients who are currently or have been recently using high-dose oral or systemic glucocorticoids, e.g. more than $7.5 \mathrm{mg}$ prednisolone or equivalent per day for 3 months or longer, should be considered for fracture risk assessment or bone mass densitometry (BMD) testing if the patient is below 40 years of age-refer to (Table 1) for detailed guidance [6].

- Audit standard 2: ACR stated that optimizing calcium intake (1000 - 1200 $\mathrm{mg} /$ day) is conditionally recommended for all patients receiving GC treatment [7]. 
Table 1. Detailed Guidance from NICE.

\section{Targeting risk assessment}

1.1 Consider assessment of fracture risk

- In all women aged 65 years and over and all men aged 75 years and over

- in women aged under 65 years and men aged under 75 years in the presence of risk factors, for example:

- previous fragility fracture

- current use or frequent recent use of oral or systemic glucocorticoids

- history of falls

- family history of hip fracture

- other causes of secondary osteoporosis

- low body mass index (BMI) (less than $18.5 \mathrm{~kg} / \mathrm{m}^{2}$ )

smoking

- alcohol intake of more than 14 units per week for women and more than 21 units per week for men.

1.2 Do not routinely assess fracture risk in people aged under 50 years unless they have major risk factors (for example, current or frequent recent use of oral or systemic glucocorticoids, untreated premature menopause or previous fragility fracture), because they are unlikely to be at high risk.

\section{Methods of risk assessment}

1.3 Estimate absolute risk when assessing risk of fracture (for example, the predicted risk of major osteoporotic or hip fracture over 10 years, expressed as a percentage).

1.4 Use either FRAX [8] (without a bone mineral density [BMD] value if a dual-energy X-ray absorptiometry [DXA] scan has not previously been undertaken) or QFracture, within their allowed age ranges, to estimate 10-year predicted absolute fracture risk when assessing risk of fracture. Above the upper age limits defined by the tools, consider people to be at high risk.

1.6 Do not routinely measure BMD to assess fracture risk without prior assessment using FRAX (without a BMD value) or QFracture.

1.7 Following risk assessment with FRAX (without a BMD value) or QFracture, consider measuring BMD with DXA in people whose fracture risk is in the region of an intervention threshold for a proposed treatment, and recalculate absolute risk using FRAX with the BMD value.

1.9 Measure BMD to assess fracture risk in people aged under 40 years who have a major risk factor, such as history of multiple fragility fracture, major osteoporotic fracture, or current or recent use of high-dose oral or high-dose systemic glucocorticoids (more than $7.5 \mathrm{mg}$ prednisolone or equivalent per day for 3 months or longer).

1.11 Take into account that risk assessment tools may underestimate fracture risk in certain circumstances, for example if a person:

- has a history of multiple fractures

- has had previous vertebral fracture(s)

- has a high alcohol intake

- is taking high-dose oral or high-dose systemic glucocorticoids (more than $7.5 \mathrm{mg}$ prednisolone or equivalent per day for 3 months or longer)

- has other causes of secondary osteoporosis.

- Audit standard 3: ACR stated that optimizing vitamin D intake (600 - 800 IU) is conditionally recommended for all patients receiving GC treatment [7].

- Audit standard 4: ACR stated that lifestyle modifications (a balanced diet, maintaining weight in the recommended range, smoking cessation, regular weight bearing or resistance training exercise, limiting alcohol intake to 1 - 2 alcoholic beverages/day) are conditionally recommended for all patients receiving GC treatment [7].

Due to local socioeconomic factors and logistic difficulties, it was decided that the audit standard be lowered to at least having $50 \%$ of patients, who met the 
audit criteria and who attended follow up clinics during the audit period, assessed and managed according to these guidelines.

\subsection{Analysis}

The data analysis was carried out using SPSS version 9.0 statistical package and Excel office 365.

\section{Results}

During the designated four week period, four rheumatology follow up clinics were attended by more than five hundred patient. Only military Insured patients were registered in the system, whereas no record can be found for noninsured patients attending the clinic, therefore the exact number of total patients cannot be specified. Forty five patients were recruited for the audit. All eligible patients received $10 \mathrm{mg}$ or more of prednisolone per day. However, the majority of non-eligible patients attending the clinic were on a daily maintenance dose of 5 mg of prednisolone with or without Disease modifying anti-rheumatic drugs (DMARDs) for their rheumatic conditions.. Most eligible patients were females (93.3\%) with BMI above $18.5 \mathrm{~kg} / \mathrm{m}^{2}$ (100\%), the age and sex distribution is shown in Figure 1. When reviewing patients follow up cards to confirm diagnoses, one patient was identified as an anomaly with a diagnosis of osteoarthritis(OA), and has been given GC for more than one year, refer for Figure 2 for patients' diagnoses. Figure 3 and Figure 4 show the different oral GC dosage and the duration of therapy among eligible patients.

Regarding other OP risk factors, thirty two patients $(71.1 \%)$ had no other risk factors for OP/OFF other than high oral GC therapy and rheumatic diseases. Of the forty two female patients taking high dose GC, two (4.4\%) were post menopausal, and seven (15.5\%) had undergone menopause before the age of 45 . Only one female patient had suffered a low trauma fracture and one patient gave a family history of OP fracture after minor trauma (Figure 5). All patients denied been housebound for long periods and they were well exposed to sun. All patients were active, denning smoking or alcohol intake. Two patients suffered recurrent falls which has not been investigated and one patient had hyperthyroidism.

Reviewing patients understanding of the OP/OFF risk with prolonged use of GC, thirty two (71.1\%) patients were not aware of the risk, and they were not well informed about the benefit of dietary modification, exercising, and sun exposure as preventive measures (Figure 6).

We performed the 10-year estimated risk assessment for major osteoporotic or hip fracture on every eligible patient, using online FRAX (GC-adjusted) assessment tool, except for patients below 40 years old where DEXA scan is recommended without prior risk assessment. DEXA scanning was performed in only three (6.7\%) patients, two of them were above 40 , had moderate risk based on FRAX tool, and only one was treated with oral bisphosphonate after con- 
firming OP. The third DEXA scan was offered to a young male patient with Bechet's disease privately in Egypt. Five (14.7\%) patients were categorized by FRAX tool to be high risk, and all were not offered DEXA or anti-resorptive therapy (Figure 7).

Medication used for prevention and management of CIOP in eligible patients consisted of calcium in all forty five (100\%) patients, calcium and vitamin D and oral bisphosphonates in one patient. No patient was receiving intravenous bisphosphonate, teriparatide, denosumab or raloxifene. Forty two (95.6\%) patients were receiving sub optimal calcium supplements with or without vitamin D.

Twenty seven $(60 \%)$ patients had an insurance that covers a quarter of their medication cost, though, they sometimes pay the full cost when medicines are not available in insurance pharmacies. Most patients admit having issues with compliance at some point either due to unaffordable cost or unavailability or both (Figure 8).

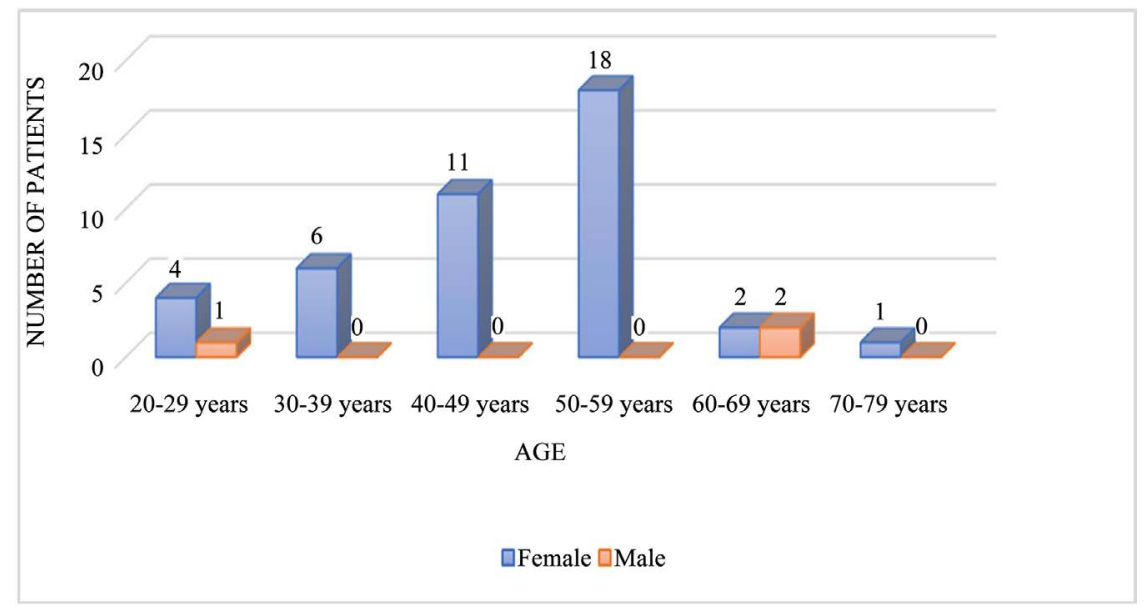

Figure1. Age and sex distribution.

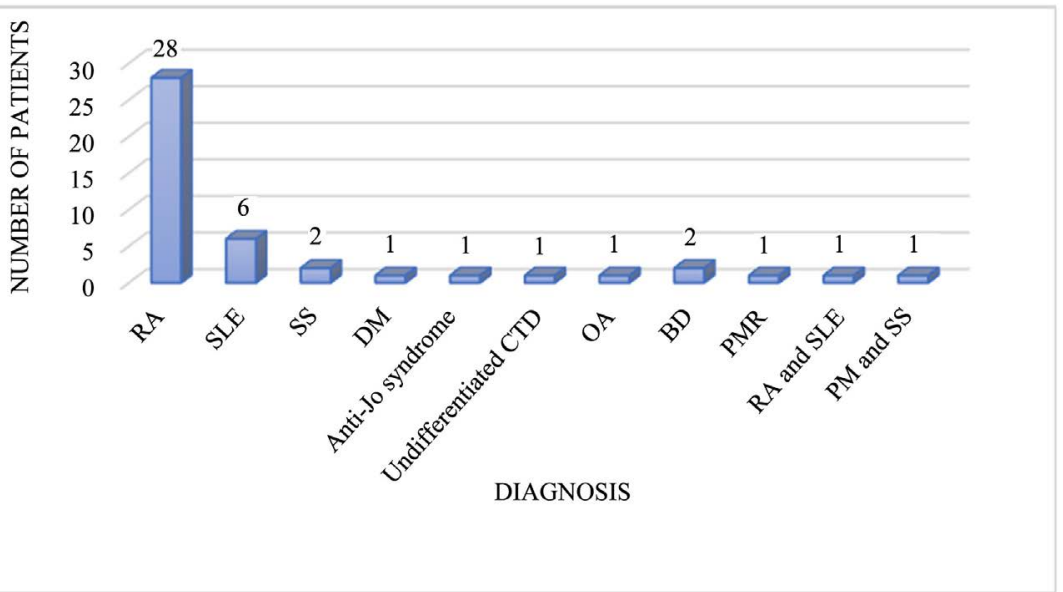

Figure 2. Rheumatic conditions among eligible patients (RA: Rheumatoid Arthritis, SLE: Systemic Lupus Erythromatosis, SS: Sjogren Syndrome, DM: Dermatomyositis, CTD: Connective tissue diseases, OA: Osteoarthritis, BD: Bechet's disease, PMR: Polymyalgia rheumatica, PM: Polymyositis. 


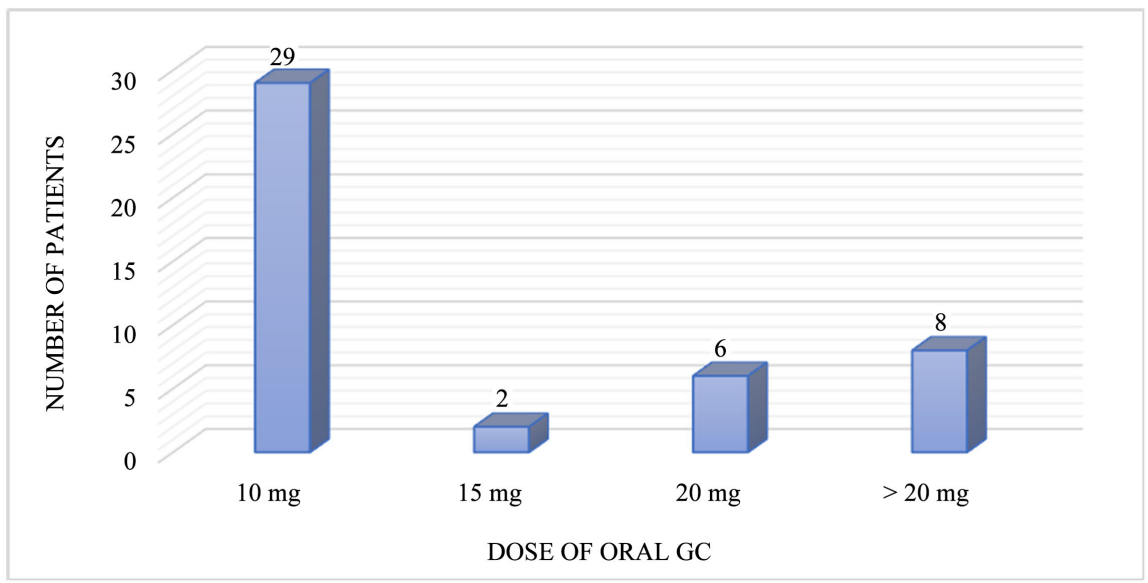

Figure 3. Different dosage of oral GC among eligible patients.

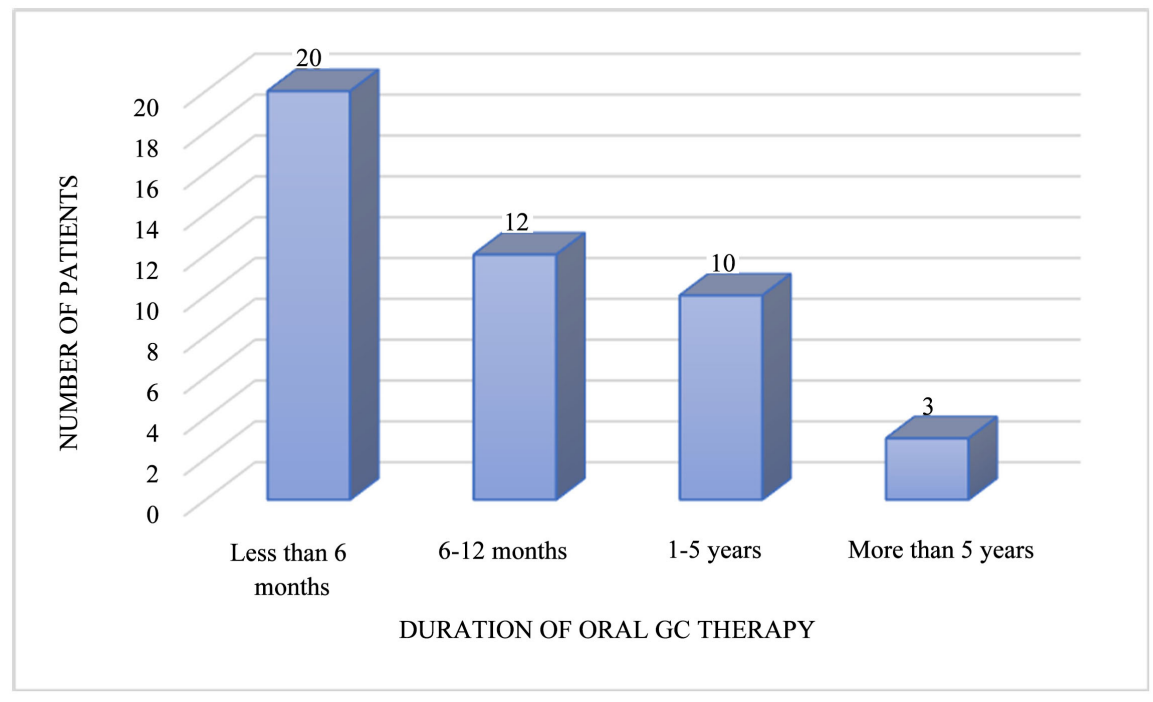

Figure 4. Duration of oral GC therapy among eligible patients.

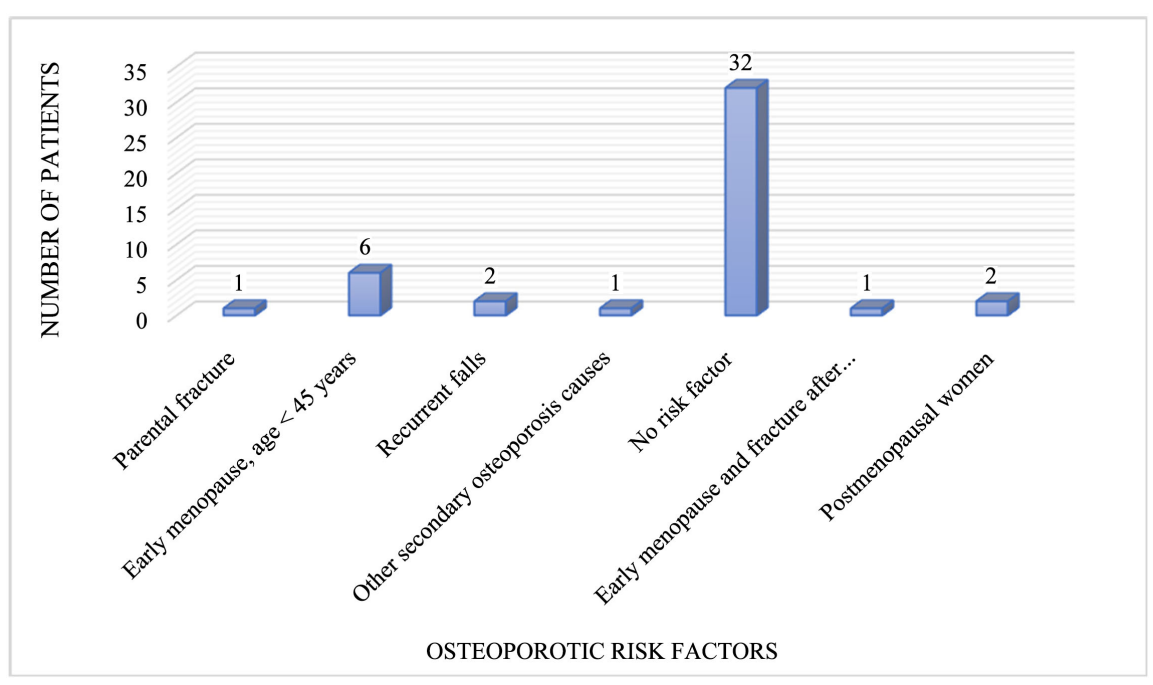

Figure 5. Distribution of other OP risk factor. 


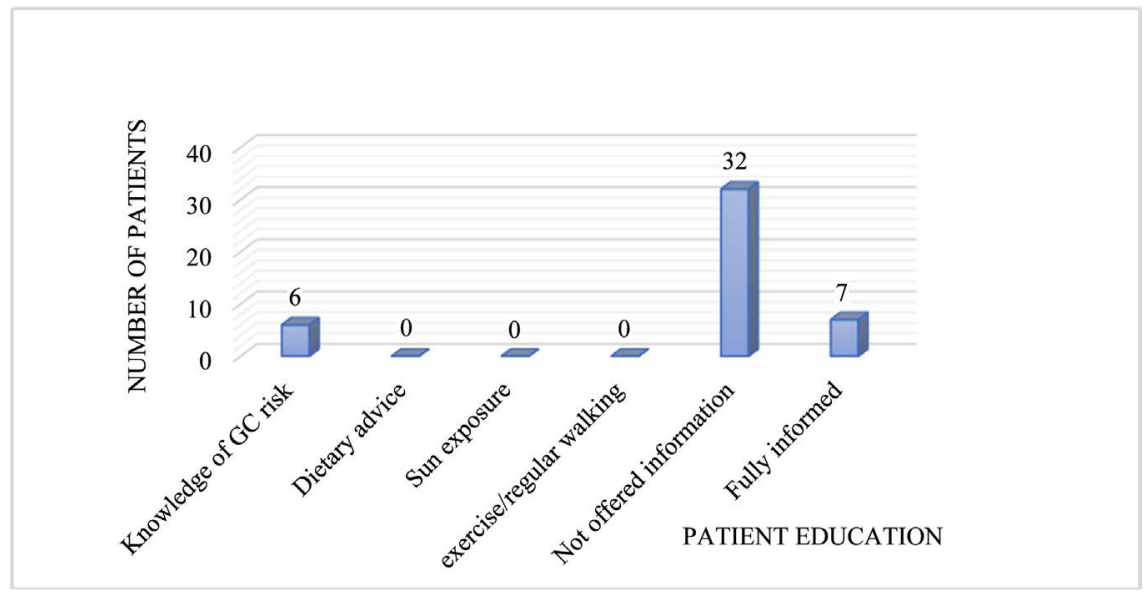

Figure 6. Patients' education.

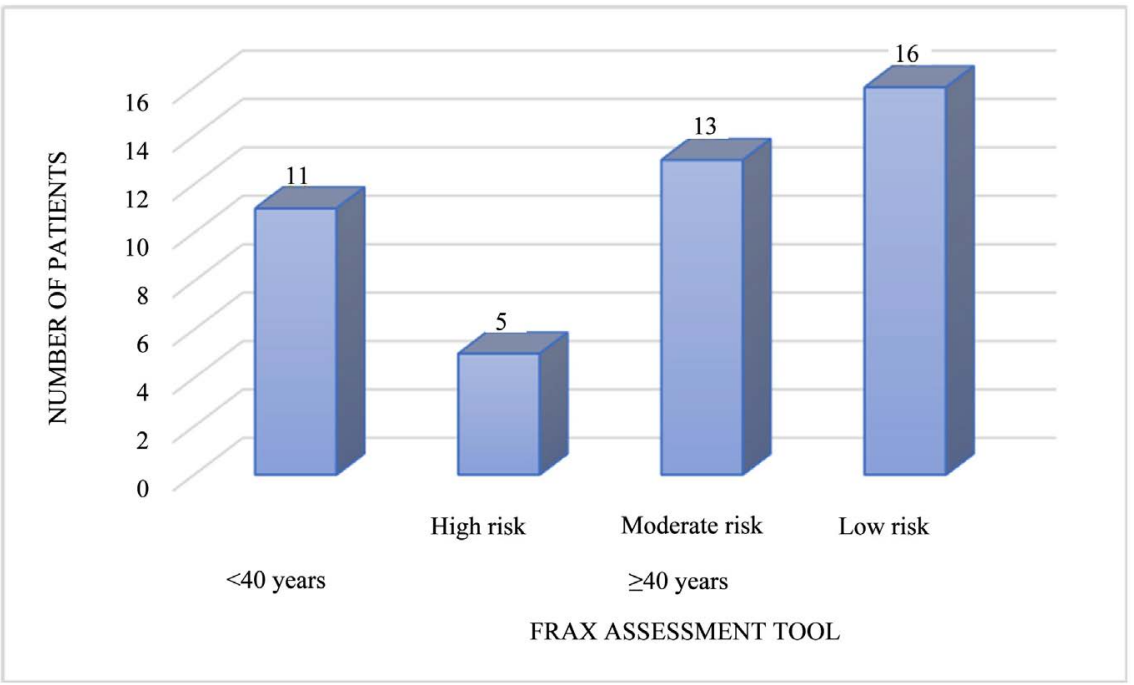

Figure 7. Age distribution and FRAX assessment in patients above 40 years old.

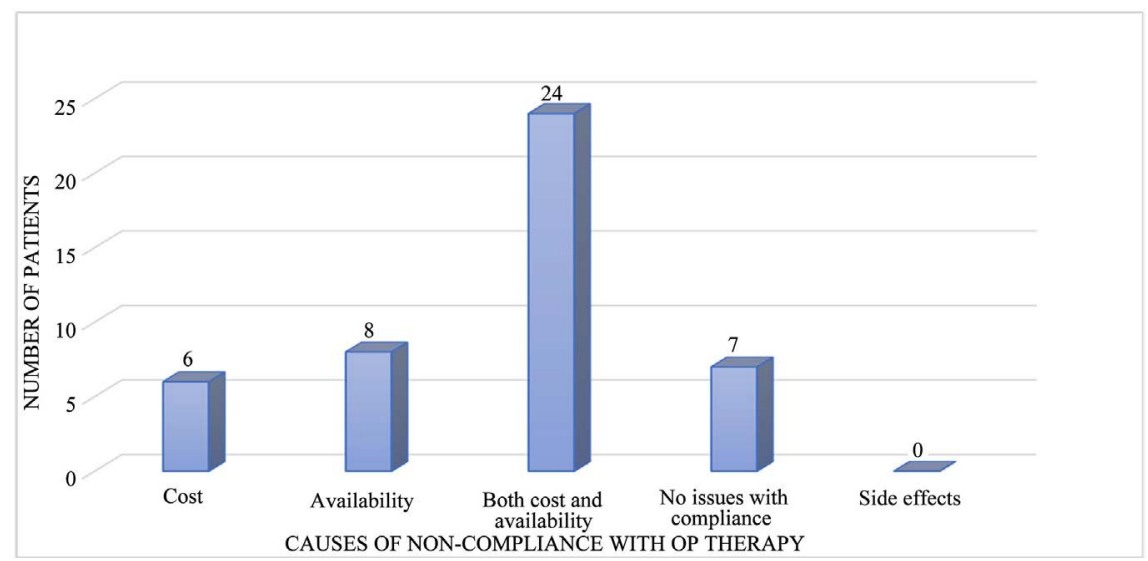

Figure 8. Causes of non-compliance with calcium with or without vitamin D.

\section{Discussion}

To our knowledge, there are few audits which looked at the risk of OP/OFF with 
long term oral GC administration in rheumatic diseases [8] [9] [10] and this is the first in Sudan. Likewise, there is no specific guidance on the topic but rather sparse recommendations that suggest considering fracture risk assessment in patients with either some rheumatic diseases (RA and SLE) or who are on long-term GC. ACR had launched Guidelines for prevention and treatment of glucocorticoid-induced osteoporosis in 2017 and it can be applicable for every steroid treated patient from other disciplines.

This audit has highlighted potential problems in the assessment of patients with rheumatic diseases on long term oral GC in the Centre. By reviewing the data on each patient against the guidelines, most patients were inappropriately undertreated (Table 2). Generally, the majority were not aware of the possible risk of OP/OFF with long term use of oral GC, which is paramount in preventing OP, because many patients self-medicate with GC as they can be obtained without prescriptions. If patient education was to be implemented when initiating GC, patients would be more vigilant. With regards to risk assessment, some patients may have been assessed for OP/OFF and deemed not suitable for BMD testing or prophylaxis bisphosphonate, but it was difficult to identify such patients based on patients' charts, therefore, we could not draw a result on audit standard 1.

Table 2. Audit results.

\begin{tabular}{|c|c|c|c|c|}
\hline Audit Standards & Target (\%) & Exception & Results & Guidelines \\
\hline $\begin{array}{l}\text { 1) adult patient who are on or } \\
\text { recently used high-dose oral or } \\
\text { high-dose systemic GC (e.g. more } \\
\text { than } 7.5 \text { mg prednisolone or } \\
\text { equivalent per day for } 3 \text { months } \\
\text { or longer) should be considered } \\
\text { for fracture risk assessment (or } \\
\text { BMD testing if patient below } 40 \\
\text { years old). }\end{array}$ & $50 \%$ & No & $\begin{array}{l}\text { Insufficient } \\
\text { information } \\
\text { to draw } \\
\text { a result. }\end{array}$ & NICE \\
\hline $\begin{array}{l}\text { 2) Optimizing calcium intake } \\
\text { (1000 - } 1200 \mathrm{mg} / \text { day) For all } \\
\text { patients receiving GC treatment. }\end{array}$ & $50 \%$ & $\begin{array}{l}\text { Causes of } \\
\text { hypercalcemia } \\
\text { and allergies to } \\
\text { calcium components. }\end{array}$ & $6.7 \%$ & ACR \\
\hline $\begin{array}{l}\text { 3) Optimizing vitamin D intake } \\
\text { ( } 600 \text { - } 800 \text { IU/day) for all patients } \\
\text { receiving GC therapy. }\end{array}$ & $50 \%$ & $\begin{array}{l}\text { Causes of } \\
\text { hypercalcemia } \\
\text { and vitamin } \\
\text { D toxicity }\end{array}$ & $\begin{array}{l}\text { Insufficient } \\
\text { information } \\
\text { to draw } \\
\text { a result. }\end{array}$ & ACR \\
\hline $\begin{array}{l}\text { 4) Optimizing lifestyle } \\
\text { modifications (a balanced diet, } \\
\text { maintaining weight in the } \\
\text { recommended range, smoking } \\
\text { cessation, regular weight bearing } \\
\text { or resistance training exercise, } \\
\text { limiting alcohol intake to } 1 \text { - } 2 \\
\text { alcoholic beverages/day ) for all } \\
\text { patients receiving GC therapy. }\end{array}$ & $50 \%$ & No & $15.6 \%$ & ACR \\
\hline
\end{tabular}


Nearly all patients received suboptimal doses of calcium with no documented calculation of daily or weekly dietary calcium intake. Some patients were tested for serum vitamin D level, but result could not be allocated for all patients due to poor record storage and documentation, therefore, no conclusion was drawn regarding optimal vitamin $\mathrm{D}$ supplementation in this group, i.e., audit standard 3. However, it is worth noting that all patients were not receiving appropriate vitamin D supplements; physician usually prescribe a specific brand, which is a combination of oral calcium and vitamin D tables (one tablet contains $500 \mathrm{mg}$ of calcium and $400 \mathrm{IU}$ of vitamin D3), but it was noticed that some physician do prescribe sub optimal dose (one tablet instead of the daily recommended dose of two as per the manufacturing company), and junior doctors may prescribe only calcium tables. We also noticed that this particular brand may not be available consistently in the market, and it may be interchangeably substituted by pure generic calcium tablets. Therefore, the exact percentage of patients taking vitamin D could not be identified. Socioeconomic data were consistent with the fact that compliance is an issue due to either high cost or unsustainable availability of medications, with local insurance covering only a quarter of total cost.

Regarding other OP risk factors, some patients had another risk factor beside their rheumatic disease and oral GC. This situation should promote assessment of fracture risk in all patients routinely in the clinic. In Sudan, fracture risk assessment tools are not widely utilized, mainly because physicians may not be aware of their function. Lack of proper OP clinic setting including electronic software, poor documentations and the overwhelming patients' flow, add to the difficulties of utilizing these tools routinely and efficiently. Storage of patient's case notes in follow up clinics is not feasible; therefore, all patients carry a summary card of their follow ups, which usually do not contain information on OP/OFF risk assessment. Physicians relay more on history taking to assess their patients rather than using a formal fracture risk assessment tool, and accordingly, they may recommend a DEXA scan or an $x$ ray if a fracture is suspected.

Most patients pay privately for their DEXA scans and OP medications, if available, which is unaffordable for the majority. Therefore, it is reasonable to consider high intensity treatment blindly (bisphosphonate) if the patient is high-risk individual. Overall, socioeconomic factors may hinder the initiation of bone protection therapy blindly or after BMD or other radiological modalities.

Recognizing these trends and other socioeconomic hardships, we anticipate that, at least, rheumatic patients on oral GC would be educated about their risk of OP and to be commenced on optimal calcium and vitamin D supplementations with lifestyle modifications. Fracture risk assessment tools are relatively simple but may underestimate risk in steroid treated patients because they do not take in to account variations in GC doses. To accurately estimate the risk associated with doses of more than $7.5 \mathrm{mg} / \mathrm{day}$, it is suggested, the clinician must multiply the risk of major osteoporotic fracture and the risk of hip fracture generated with the FRAX by 1.15 and by 1.2 , respectively [7], then if patients are at 
moderate to high risk, they should be referred for BMD testing, or to be commenced on bisphosphonate without prior BMD testing especially when there is history of OFF, or if the patient has multiple major risk factors.

We acknowledge that the burden of GIOP has a far reaching impact on patients with rheumatic diseases in a way which cannot be measured in Sudan. We feel that many patients with rheumatic diseases who were on long term GC have already suffered from OFF and destined to be bound to their homes, not being able to attend their medical follow ups, brought to hospitals only when they are sick, in contrast to mobile patients who can still attend their appointments. Therefore, the audit is largely biased by the fact that we cannot have access to patients who truly suffer from the consequences of this practice.

On the other hand and under current circumstances, it would be unreasonable to recommend rheumatologist to reduce their overall prescriptions of GC; Disease modifying anti-rheumatic drugs (DMARDS), apart from Methotrexate and Hydroxychloroquine, are constantly unavailable in the market for weeks or even months, therefore, physicians are obliged to continue steroids for longer periods of time for convenience, as a substitute, in patients who require treatment with more than two DMARDS. The use of biologics in Sudan is still a new arena, limited by cost.

We conclude that physician should maximize their patients' counselling and prevention of OP and develop a clear plan on starting prophylaxis bisphosphonate without prior DEXA scan on high risk individual. At the time of conducting this audit, only one machine was functional in a private hospital in Khartoum. Currently efforts are made by dedicated physicians to include alendronate under local insurance umbrella for a reasonable price.

\section{Recommendations}

Recommendations were set to improve the awareness of physicians as well as patients on the risk of OP and OFF with long term oral GC treatments, and to increase the rate of counseling and management of OP and OP risk factors in steroid treated patients.

- All patients taking (or who are likely to take) $7.5 \mathrm{mg}$ or more a day of prednisolone (or equivalent) for three months or more:

1) Should be assessed for their risk of fragility fracture using fracture risk assessment tools if applicable and the result should be documented. Physicians my want to use online tools such as FRAX to calculate their patients risk.

2) Should be given

- Advice regarding GC side effects including the risk of OP/OFF and other risk factors for OP/OFF.

- Dietary advice (especially adequate calcium and vitamin D): calcium intake should be calculated and documented in patient's chart. It is always advice to check and document serum vitamin $\mathrm{D}$ levels first and manage accordingly.

- Advice regarding regular weight-bearing exercise/walking. 
- Advice regarding maintenance of body weight.

- Smoking status and appropriate intervention.

- Alcohol use and appropriate intervention.

- Assessment of falls risk and advice if appropriate.

- Advice regarding the importance of sun exposure.

3) Patients may be referred for BMD testing or radiological imaging such as vertebral imaging if OFF is suspected or if the treating physician feels the patient is a high-risk individual for $\mathrm{OP}$ in the presence of long term high dose oral GC administration and other independent or major risk factors.

4) Should be on optimal calcium and vitamin D supplements as stated above.

5) Physicians may consider starting bisphosphonate without prior BMD testing in selected individuals.

Note: Physician may want to apply these recommendations in all steroid treated patients who are on long term therapy for their rheumatic diseases (e.g., $5 \mathrm{mg}$ of prednisolone for 3 months or more).

\section{Interventions and Reauditing}

Authors felt that the most suitable way to implement these recommendations and promote understanding of the topic, was to prepare educational materials in the form of posters for both patients and doctors to be available in outpatient clinics with planning to re audit after one year.

\section{Ethical Consideration}

Ethical approval was obtained from appropriate authorities.

\section{Acknowledgements}

We would like to thank Dr Mashaar Osama for performing the data analysis.

\section{Conflicts of Interest}

The authors declare no conflicts of interest regarding the publication of this paper.

\section{References}

[1] Curtis, J.R., Westfall, A.O., Allison, J., Bijlsma, J.W., Freeman, A., George, V., Kovac, S.H., Spettell, C.M. and Saag, K.G. (2006) Population-Based Assessment of Adverse Events Associated with Long-Term Glucocorticoid Use. Arthritis Care \& Research, 55, 420-426. https://doi.org/10.1002/art.21984

[2] Laan, R.F., van Riel, P.L., van de Putte, L.B., Leon, J.T., van't Hof, M.A. and Lemmens, J.A. (1993) Low-Dose Prednisone Induces Rapid Reversible Axial Bone Loss in Patients with Rheumatoid Arthritis: A Randomized, Controlled Study. Annals of Internal Medicine, 119, 963-968. https://doi.org/10.7326/0003-4819-119-10-199311150-00001

[3] Cortet, B., Flipo, R.M., Blanckaert, F., Duquesnoy, B., Marchandise, X. and Delcambre, B. (1997) Evaluation of Bone Mineral Density in Patients with Rheumatoid 
Arthritis. Influence of Disease Activity and Glucocorticoid Therapy. Revue du rhumatisme (English ed.), 64, 451-458.

[4] Laan, R.F., Buijs, W.C., Verbeek, A.L., Draad, M.P., Corstens, F.H., Van de Putte, L.B. and Van Riel, P.L. (1993) Bone Mineral Density in Patients with Recent Onset Rheumatoid Arthritis: Influence of Disease Activity and Functional Capacity. Annals of the Rheumatic Diseases, 52, 21. https://doi.org/10.1136/ard.52.1.21

[5] Cortet, B., Guyot, M.H., Solau, E., Pigny, P., Dumoulin, F., Flipo, R.M., Marchandise, X. and Delcambre, B. (2000) Factors Influencing Bone Loss in Rheumatoid Arthritis: A Longitudinal Study. Clinical and Experimental Rheumatology, 18, 683-690.

[6] O’Flynn, N. (2012) Risk Assessment of Fragility Fracture: NICE Guideline. British Journal of General Practice, 62, 667-668. https://doi.org/10.3399/bjgp12X659475

[7] Buckley, L., Guyatt, G., Fink, H.A., Cannon, M., Grossman, J., Hansen, K.E., Humphrey, M.B., Lane, N.E., Magrey, M., Miller, M. and Morrison, L. (2017) American College of Rheumatology Guideline for the Prevention and Treatment of Glucocorticoid-Induced Osteoporosis. Arthritis Care \& Research, 69, 1095-1110. https://doi.org/10.1002/acr.23279

[8] Erb, N., Duncan, R.C., Raza, K., Rowe, I.F., Kitas, G.D. and Situnayake, R.D. (2002) A Regional Audit of The Prevention and Treatment of Corticosteroid-Induced Osteoporosis in Patients with Rheumatic Diseases in the West Midlands. Rheumatology, 41, 1021-1024. https://doi.org/10.1093/rheumatology/41.9.1021

[9] Srinivasulu, N., Sharma, V., Chitnis, N., Mangat, G., Samant, R. and Canchi, B. (2010) Primary Prophylaxis for Steroid-Induced Osteoporosis: Are We Doing Enough?-An Audit from A Tertiary Care Centre. Indian Journal of Rheumatology, 5, 176-179. https://doi.org/10.1016/S0973-3698(11)60005-8

[10] Walker-Bone, K., Wood, A., Hull, R., Ledingham, J.M., McCrae, F.C., Shaban, R., Thomas, A. and Mackay, K. (2004) The Prevention and Treatment of Glucocorticoid-Induced Osteoporosis in Clinical Practice. Clinical Medicine, 4, 431-436. 